\title{
Muséologies
}

Les cahiers d'études supérieures

\section{Archéologie du numérique}

\section{Alessandra Mariani}

Volume 6, numéro 2, 2013

La cybermuséologie

URI : https://id.erudit.org/iderudit/1018934ar

DOI : https://doi.org/10.7202/1018934ar

Aller au sommaire du numéro

Éditeur(s)

Association Québécoise de Promotion des Recherches Étudiantes en

Muséologie (AQPREM)

ISSN

1718-5181 (imprimé)

1929-7815 (numérique)

Découvrir la revue

Citer ce compte rendu

Mariani, A. (2013). Compte rendu de [Archéologie du numérique]. Muséologies, 6(2), 132-141. https://doi.org/10.7202/1018934ar

Tous droits réservés (C Association Québécoise de Promotion des Recherches Étudiantes en Muséologie (AQPREM), 2013
Ce document est protégé par la loi sur le droit d'auteur. L’utilisation des services d'Érudit (y compris la reproduction) est assujettie à sa politique d'utilisation que vous pouvez consulter en ligne.

https://apropos.erudit.org/fr/usagers/politique-dutilisation/ 


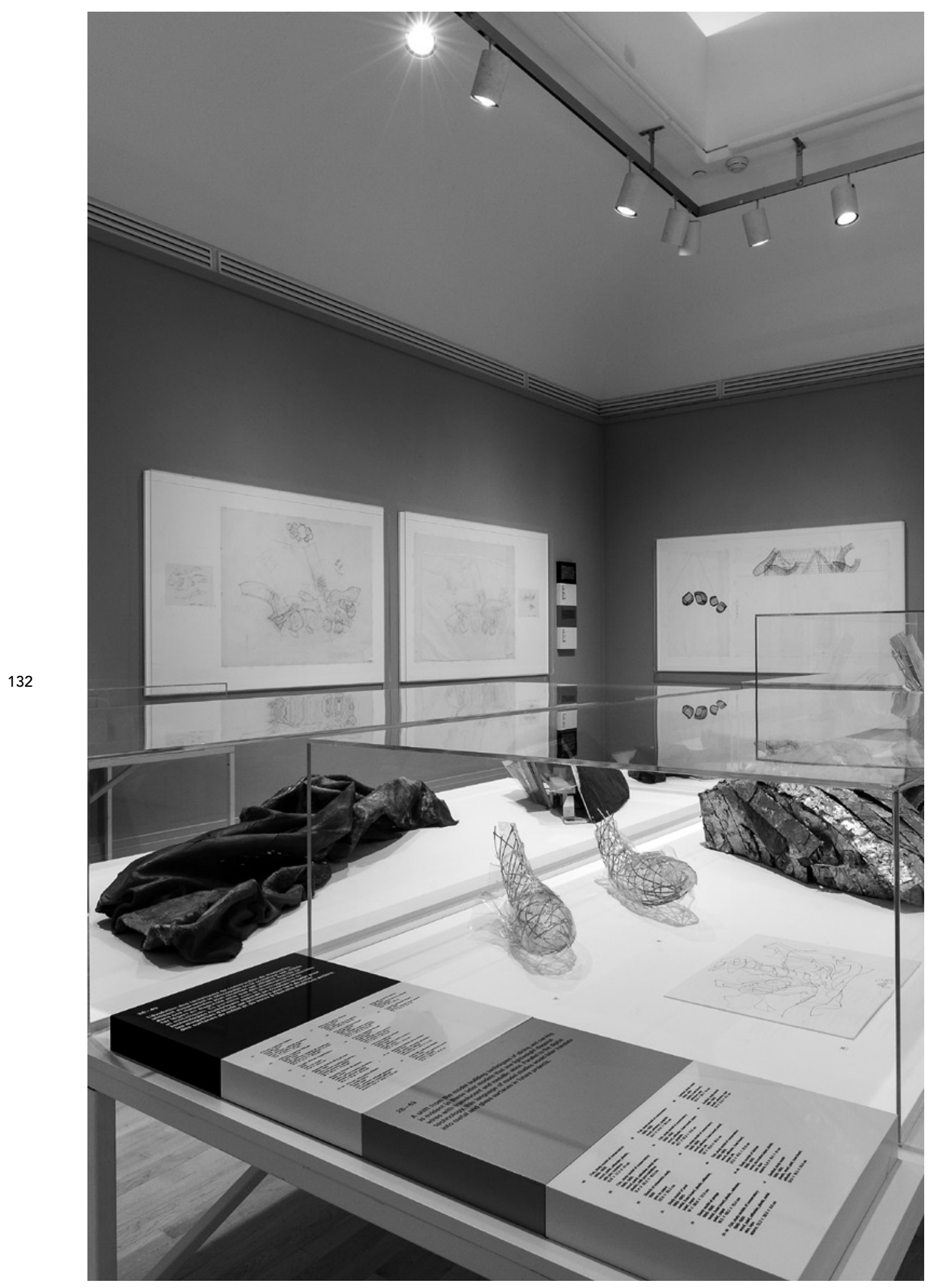

Vue de l'exposition, Archéologie du numérique, 2013.

(c) CCA, Montréal. 
Carnet

Archéologie du numérique

Alessandra Mariani

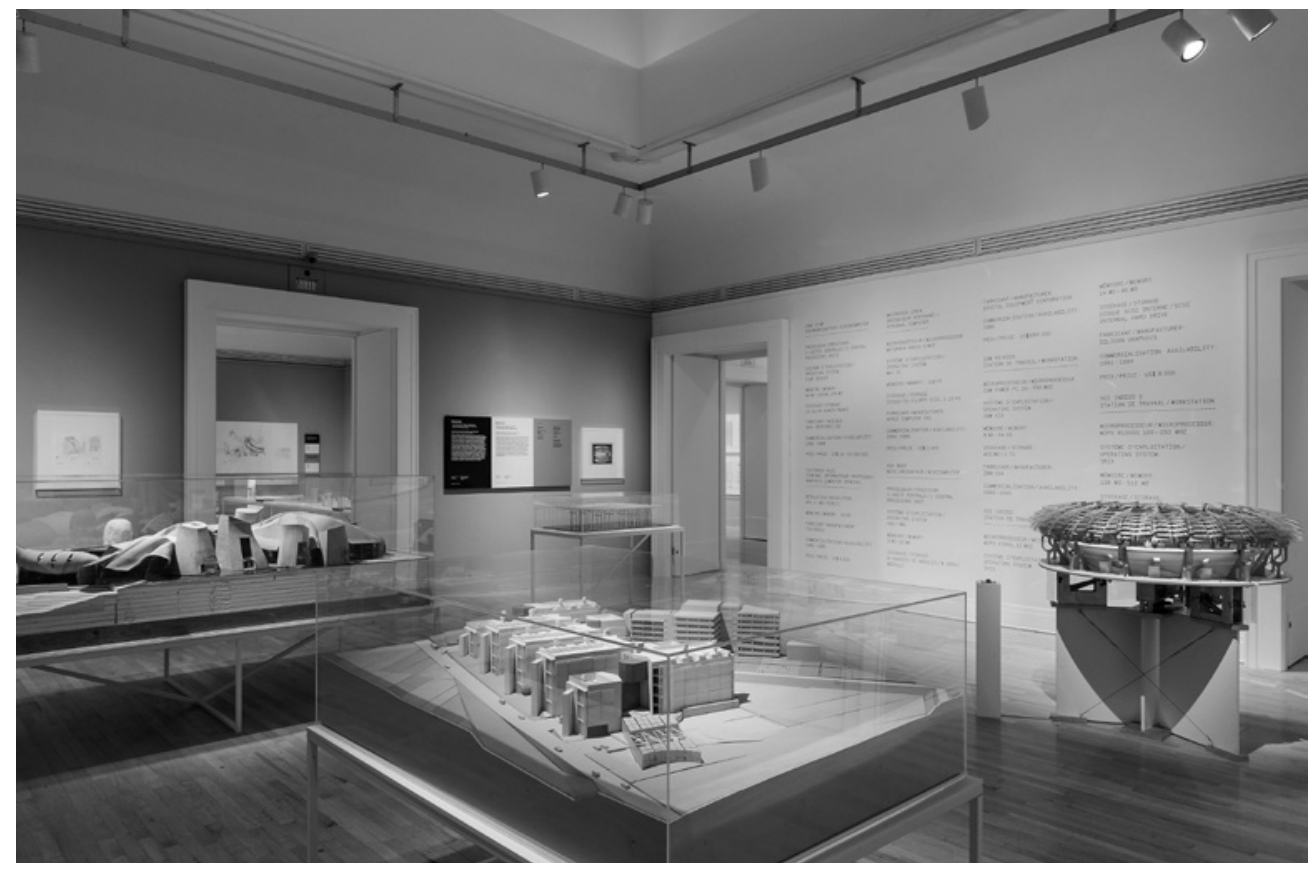

Vue de l'exposition, Archéologie du numérique, 2013.

(c) CCA, Montréal. 


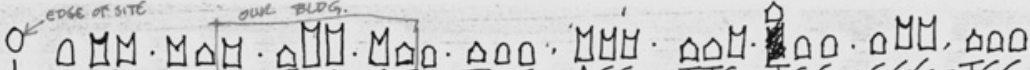
CGA- ATG - TGG-ATC-TCC-AGG - TTC-TCC-CGG-TCC -

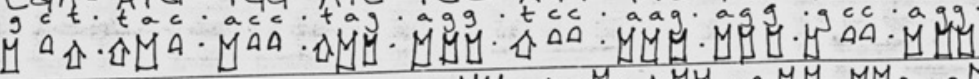
MU. C 2 C

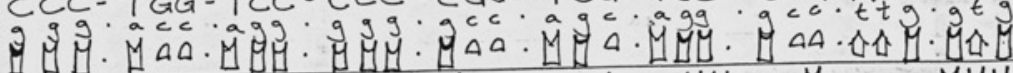

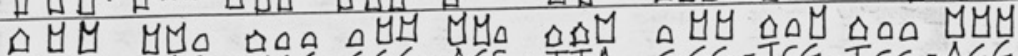
$T G G-G A C-T C C-C G G-A G C-T T A-C G G-T C G-T C C-A G G$

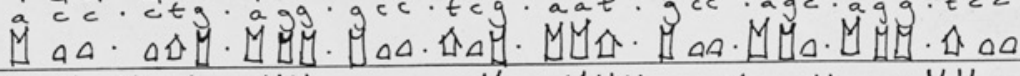

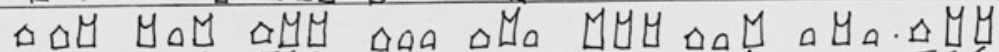
$T T G \cdot G C A-T G G-T C C-T A C-A G G-T C A-C G C-T G G$ a a $c \cdot c g t \cdot a c c \cdot a g g \cdot a t g \cdot t c c \cdot a g t \cdot g c g \cdot a c c \cdot$

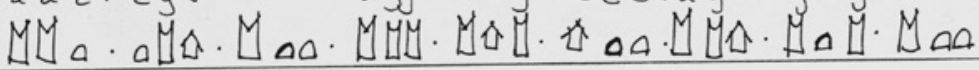

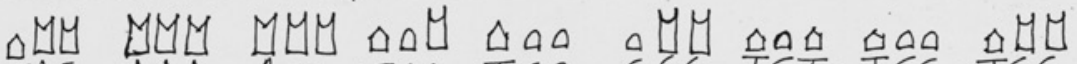
$T A G-A A A-A G G-T C G-T C C-C G G-T C T-T C C-T G G$

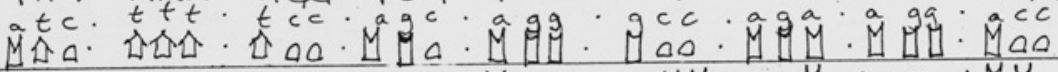

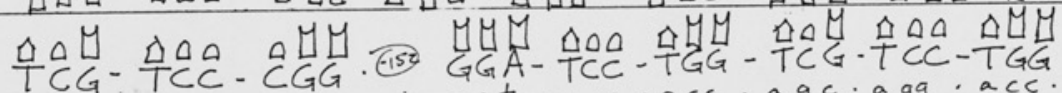
a $c$. agg. gcc. cct. agg. acc. a gc. a gg. acc.

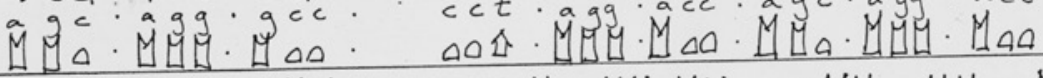

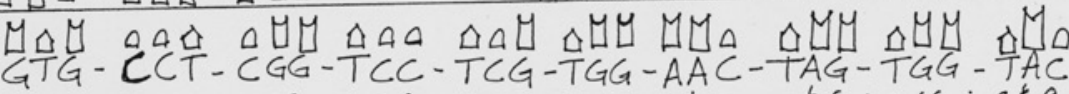

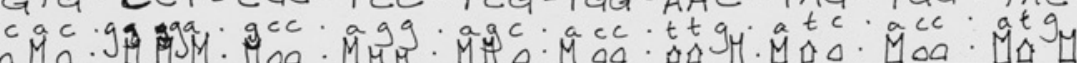

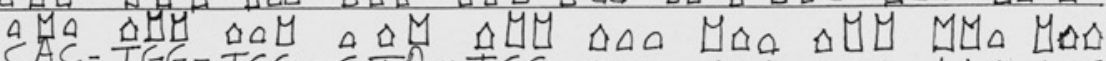
$C A C-T G G-T C G-C \frac{1}{A}-\frac{1}{C G G}-T C C-G T C-T G G-A A C-G T T$.

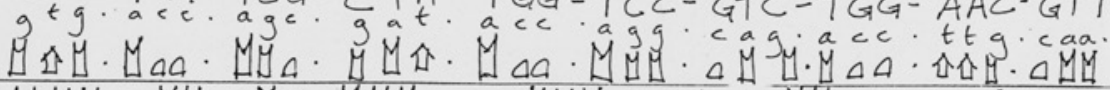

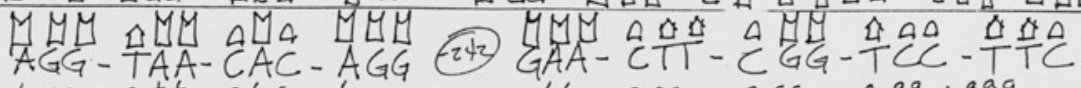
$t c c$. a t $t$. $g \in g$. $t c c$.

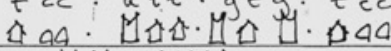

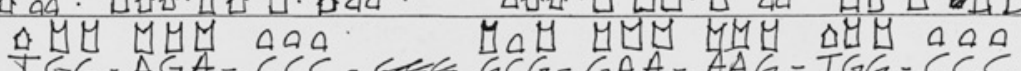
$T G G-A G A-C C C-G C G-G A A-A A G-T G G-C C C$

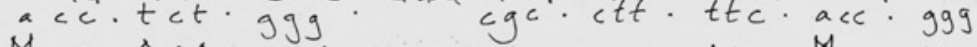
M $\Delta \Delta \cdot \Delta \Delta \cdot$ MUL.

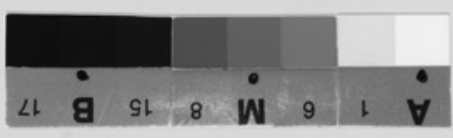

Peter Eisenman, Eisenman/Robertson Architects. Biozentrum, Centre de biologie de l'université

J.W. Goethe, Francfort-sur-le-Main, Allemagne : représentation schématique d'une séquence ADN, 1987. Peter Eisenman fonds, Centre Canadien d'Architecture. Impression électrostatique sur papier avec annotations au stylo, $28 \times 21,5 \mathrm{~cm}$. 
Le Centre Canadien d'Architecture présente du 7 mai au 13 octobre 2013 l'exposition L'Archéologie du numérique, conçue par Greg Lynn, architecte et professeur, spécialiste de la conception des formes fonctionnelles et ergonomiques générées par la technologie numérique. Ce premier volet d'une série de trois expositions sur le sujet vise à faire le point sur la perception et les attentes que nous entretenons face aux technologies et à l'environnement numérique. L'exposition est à la fois la mise en montre d'une collection en voie de se constituer ${ }^{1}$ et une réflexion sur la nature et l'impact des technologies numériques, tant dans les processus d'idéation que dans ceux de la réalisation de l'architecture. L'objectif de ce premier volet est de désamorcer le mythe utopique créé autour de ces nouveaux dispositifs de représentation et de lire dans une trame historique les projections faites autour de ceux-ci. Par cette série d'expositions, Lynn souhaite réévaluer et corriger la présomption commune qui fait de l'architecture une discipline assujettie à l'environnement numérique, afin de démontrer que la transformation des deux champs s'est réalisée de façon corrélative.

Le récit de l'exposition est imagé autour de la production de quatre projets qui permettent de présenter la variété de la recherche, l'exploration et l'application des technologies de la fin des années 1980 au début des années 1990. Selon Lynn, ces projets ont été retenus tant pour leur impact théorique dans le discours sur l'environnement numérique et l'architecture, que pour leur caractère opérationnel. La résidence Lewis de Frank Gehry (1985-1995), le Biozentrum de Peter Eisenman (1987), les sphères déployables de Chuck Hoberman (1992), la structure de la toiture du Complexe sportif municipal d'Odawara (1991) et de la Galaxy Toyama (1992) de Shoei Yoh ont tous été retenus parce qu'ils véhiculaient une valeur emblématique d'ordre cognitif, conceptuel et esthétique. Chacun de ces quatre projets comportait une intentionnalité que les concepteurs avaient développée sur la base d'un ensemble d'assomptions, expérimentant avec les logiciels disponibles ou les développant eux-mêmes, afin de pouvoir directement les appliquer dans la phase d'élaboration des projets et d'atteindre leurs objectifs. Lynn croit en outre que l'architecture n'a pas seulement mis en œuvre sa numérisation; elle aurait aussi contribué au développement de l'environnement numérique au sens plus large. Les quatre visions et trajectoires très distinctes qui sont regroupées dans l'exposition en font sa force, parce qu'elle parvient à représenter de façon assez globale la variété des médiums, des mesures et des directions pris tout au long de l'élaboration des technologies qui deviendront ultérieurement les outils usuels de l'architecture.

Eisenman cherchait dans le numérique toutes les itérations des codes qu'il écrivait; il employait la technologie comme un instrument de cryptage linguistique. Ses dessins présentés dans la première salle

1 Le directeur du CCA, Mirko Zardini, a annoncé le développement d'un chantier de recherche important sur le numérique au CCA et celui de la constitution d'une collection représentative d'un moment déterminant de la pensée architecturale, soit celui de sa numérisation. 


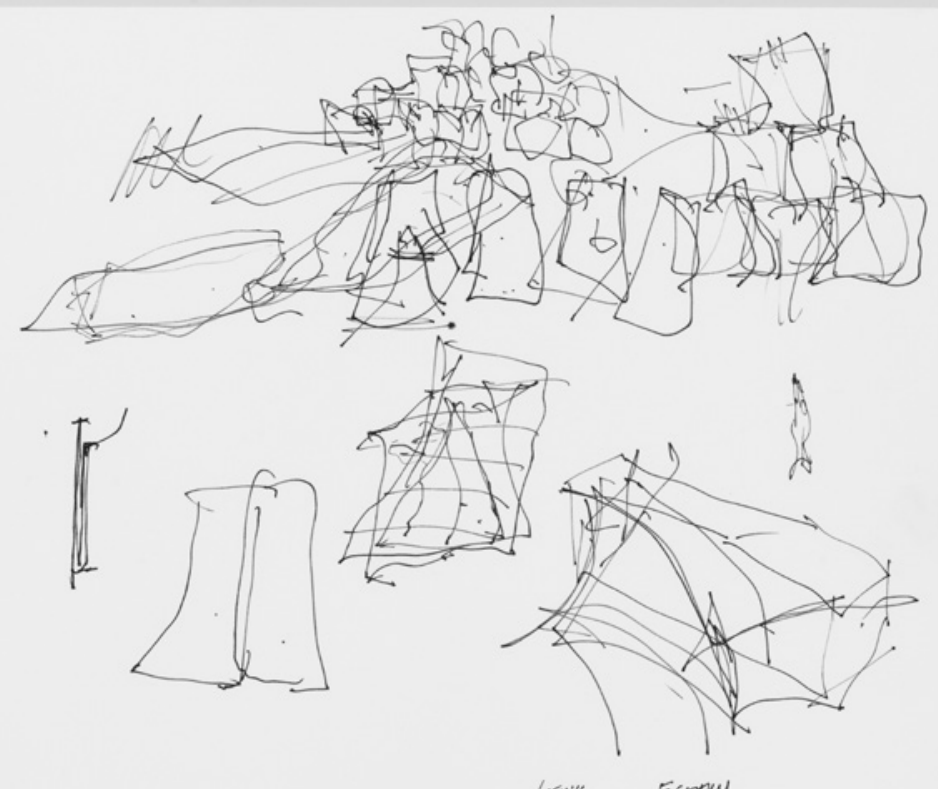

Frank O. Gehry \& Associates, Inc. Résidence Lewis, Lyndhurst, Ohio : Croquis d'études volumétriques, 1989-1995.

Image fournie par Gehry Partners, LLP.

Stylo sur papier, $22,86 \times 30,48 \mathrm{~cm}$. 


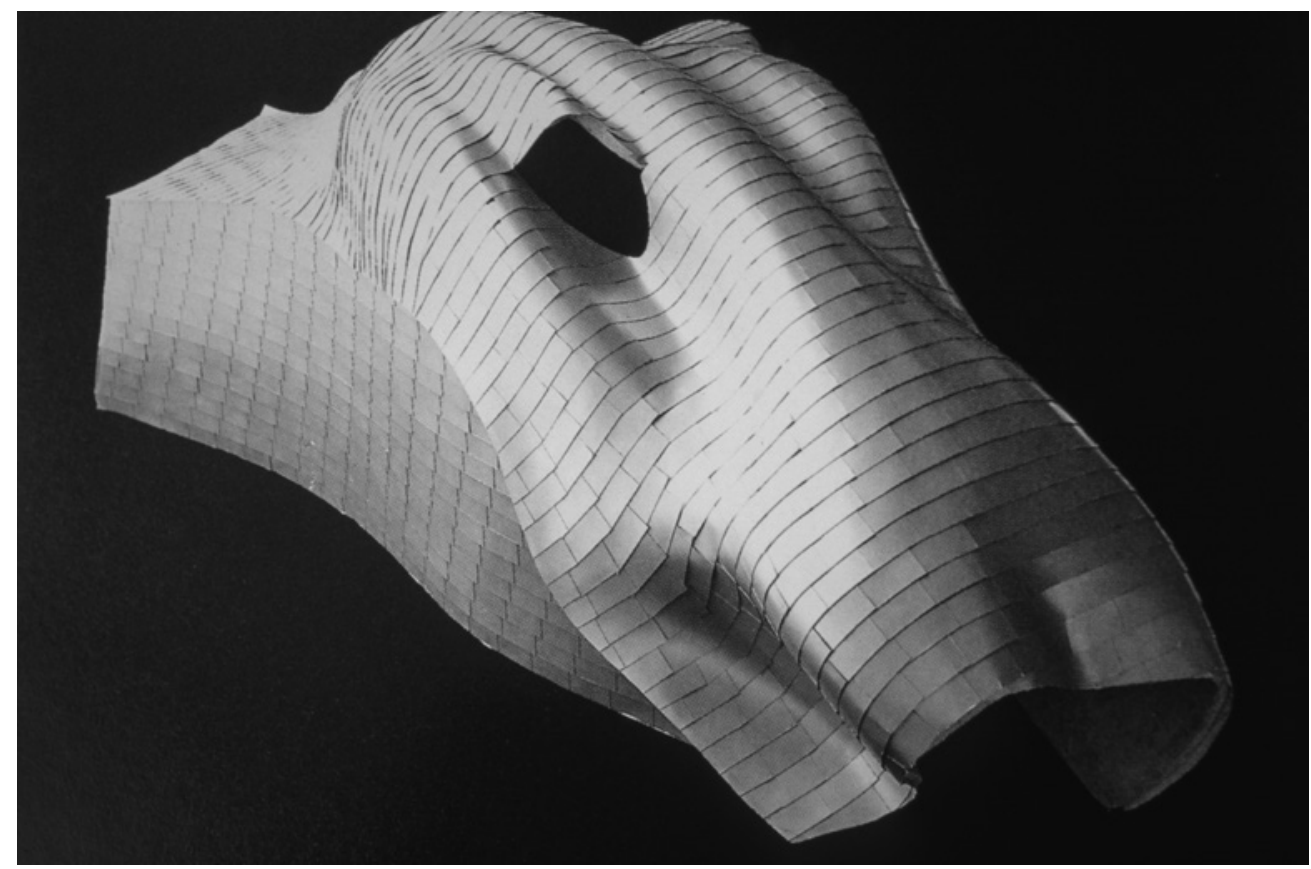

Frank O. Gehry \& Associates, Inc. Résidence Lewis, Lyndhurst, Ohio: Rendu en élévation fait à partir d'une maquette 3D Catia, 1989-1995.

Image fournie par Gehry Partners, LLP.

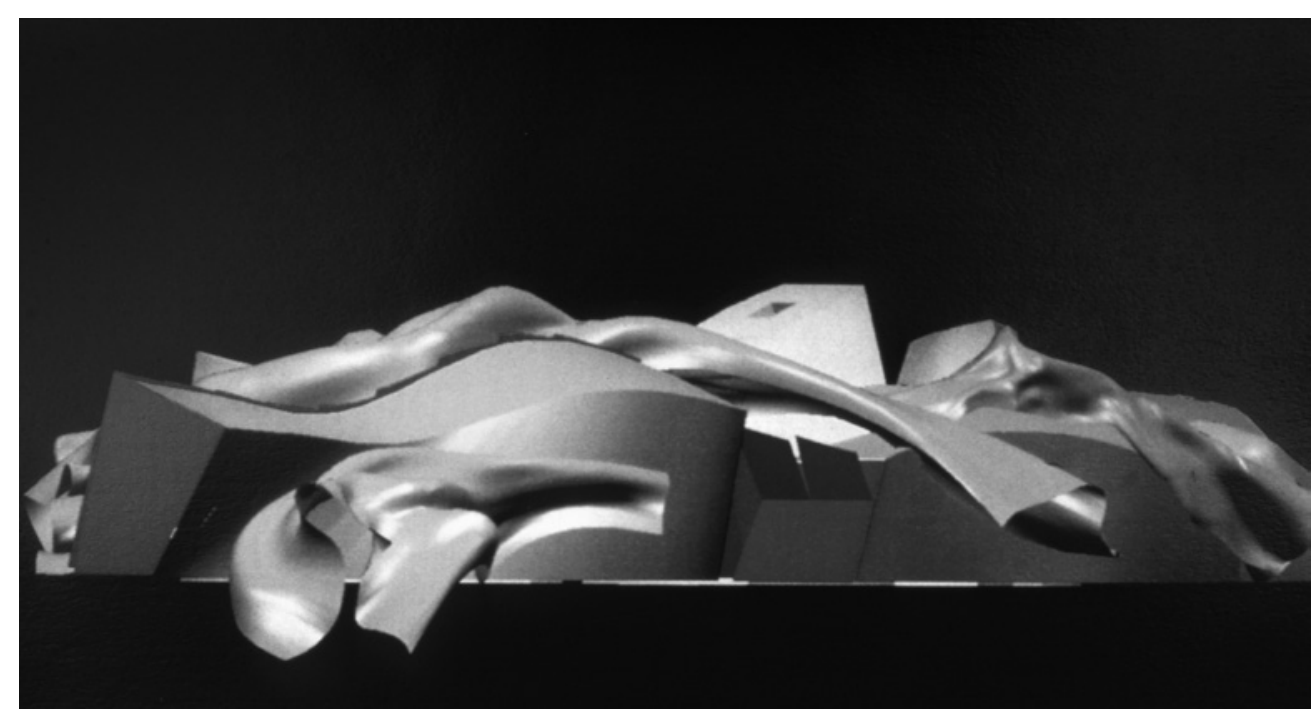

Frank O. Gehry \& Associates, Inc. Résidence Lewis, Lyndhurst, Ohio: Rendu en élévation fait à partir d'une maquette 3D Catia, 1989-1995.

Image fournie par Gehry Partners, LLP. 


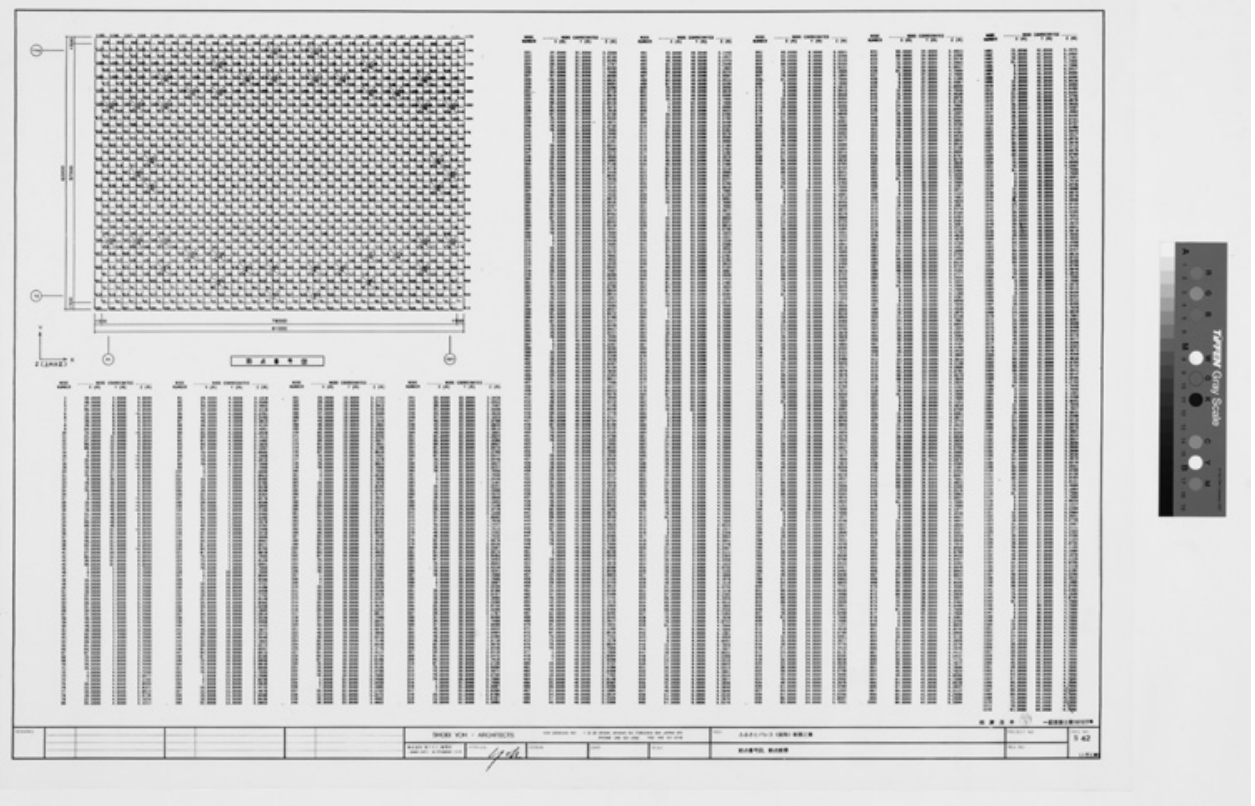

Shoei Yoh + Architects. Galaxy Toyama, gymnase, Imizu, Toyama, Japon : Plan schématique du toit avec coordonnées des nœuds, 1990-1992.

Shoei Hamura fonds, Centre Canadien d' Architecture.

(c) Shoei Yoh + Architects. Impression électrostatique sur papier translucide, 59,6 x 83,8 cm @ Shoei Yoh + Architects

de l'exposition démontrent son acharnement à les arrimer au langage de l'ordinateur; ils illustrent aussi comment, à cette époque, la vitesse de la technologie numérique allait de pair avec la technologie analogique. Les projets de Yoh sont représentés par des maquettes et des photographies de ces dernières qui révèlent leurs modèles structuraux. Des études photo-élastiques de modèles acryliques qui exhibent ces mêmes modèles et des études en format numérique de forces et de tensions diverses viennent compléter l'illustration de ce processus. Sa recherche vient associer une considération pour les éléments naturels (tels que l'éclairage des surfaces et le poids de l'accumulation de la neige) à l'efficacité économique. L'ordinateur devient ainsi un outil pour aboutir à des effets formels issus de phénomènes. Dans le cas de Hoberman, les dessins à main levée sont présentés avec la traduction codée de ces derniers à des fins de transformations géométrique et numérique culminant dans une dynamisation de l'architecture. Le mouvement de va-et-vient récurrent et commun aux protagonistes de l'exposition (soit le passage des croquis à leur transcription en signes, leur transfert ultérieur vers la numérisation et le suivi mutuel de ces processus) est annonciateur de la transformation de la technologie numérique architecturale. II s'agirait, selon Lynn, de la première ins- 
tance où la technologie aurait démontré sa potentialité à radicalement changer la nature statique et stable des bâtiments vers une perspective de robotisation et de dynamisme. Dans la maison Lewis conçue par Gehry, l'ordinateur devient un outil d'expression formelle et spatiale. Ce cas démontre comment la numérisation des modèles par ordinateur entreprise au tout début du projet est ensuite reprise par son auteur dans ses esquisses manuelles, comme s'il cherchait un corollaire à l'expression numérique. L'exécution de la rationalisation de l'expression formelle recherchée par ce dernier débouchera sur la découverte de la modélisation de l'imagerie numérique, qui évoluera par la suite en une stylisation formelle.

Riche de maquettes, de dessins et d'artéfacts divers, l'exposition se clôt avec l'appareillage responsable de ces découvertes (sauf bien entendu les ordinateurs de première génération qui auraient requis un espace indisponible au (CA) et les premiers manuels à l'usage des programmeurs et des architectes. Si représenter l'architecture de façon tangible est une opération difficile (maquettes, plans, extraits filmiques, archives variées ne suffisent jamais à reproduire l'entité physique), on peut déduire que la difficulté est exacerbée lorsqu'elle est déployée dans son format numérique. La limpidité de cette exposition vient toutefois contredire quelque peu cet énoncé; chaque salle étale son contenu et crée un rapport conséquent avec la suivante. Si toutes

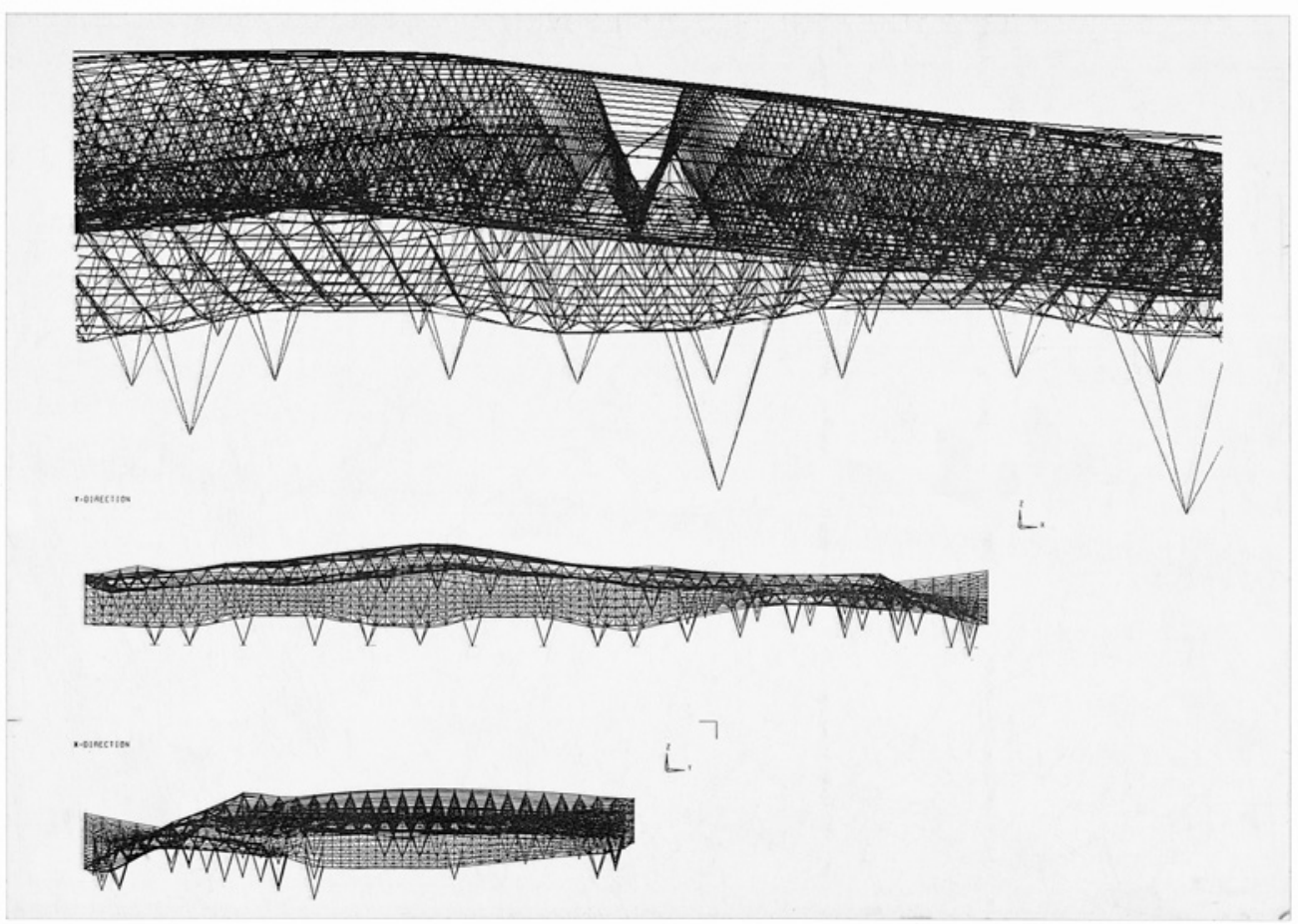

Shoei Yoh + Architects. Complexe sportif municipal, Odawara, Kanagawa, Japon : Images générées par ordinateur de la déformation du toit, 1990-1991. (C) Shoei Yoh + Architects. Impression électrostatique sur plastique, $25,7 \times 36,4 \mathrm{~cm}$ 


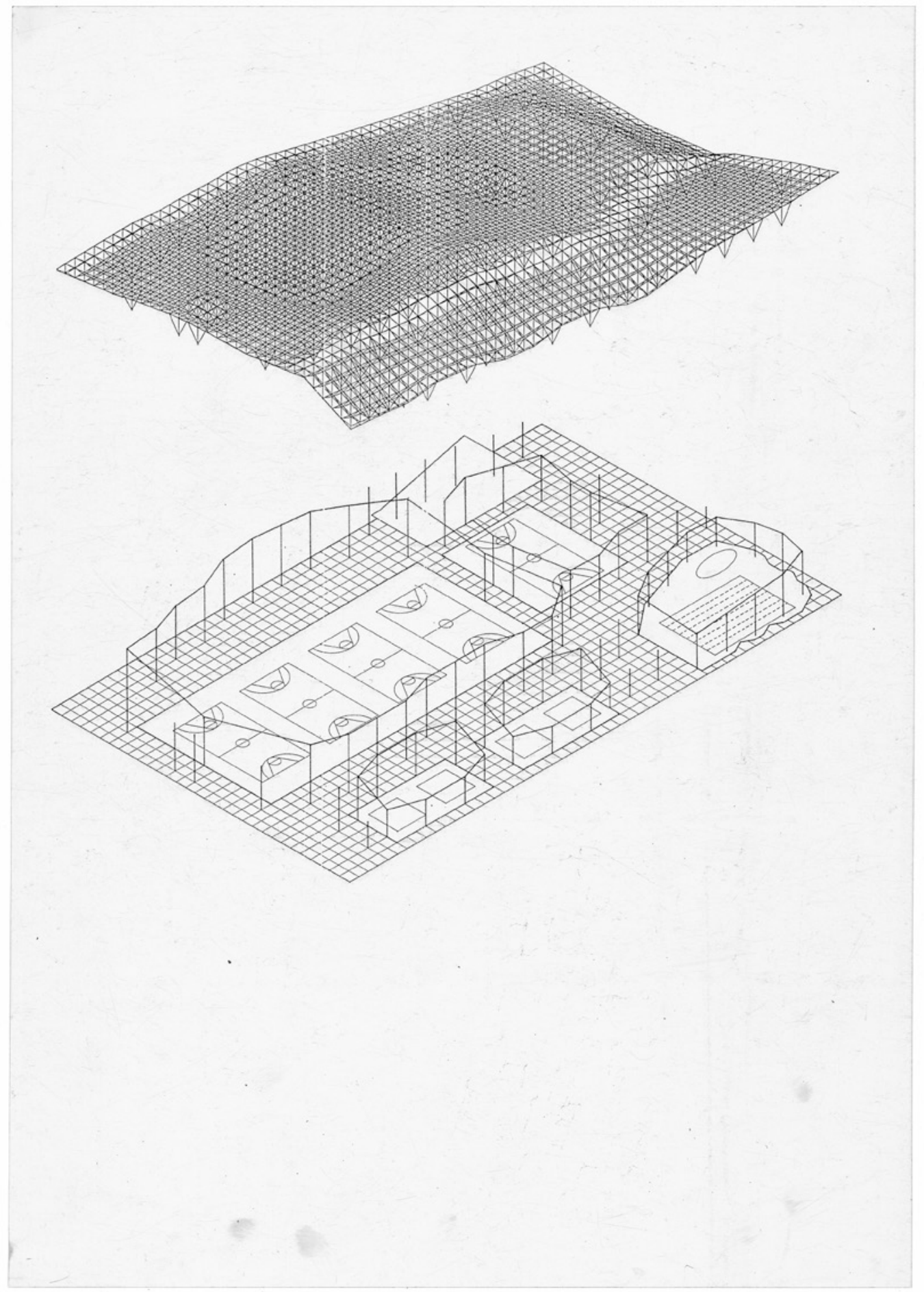

Shoei Yoh + Architects. Complexe sportif municipal, Odawara, Kanagawa, Japon: Vue éclatée montrant la conception du toit en relation avec les fonctionsintérieures, 1990 1991. Shoei Hamura fonds, Centre Canadien d'Architecture.

(C) Shoei Yoh + Architects. Impression électrostatique sur plastique, $25,7 \times 36,35 \mathrm{~cm}$. 


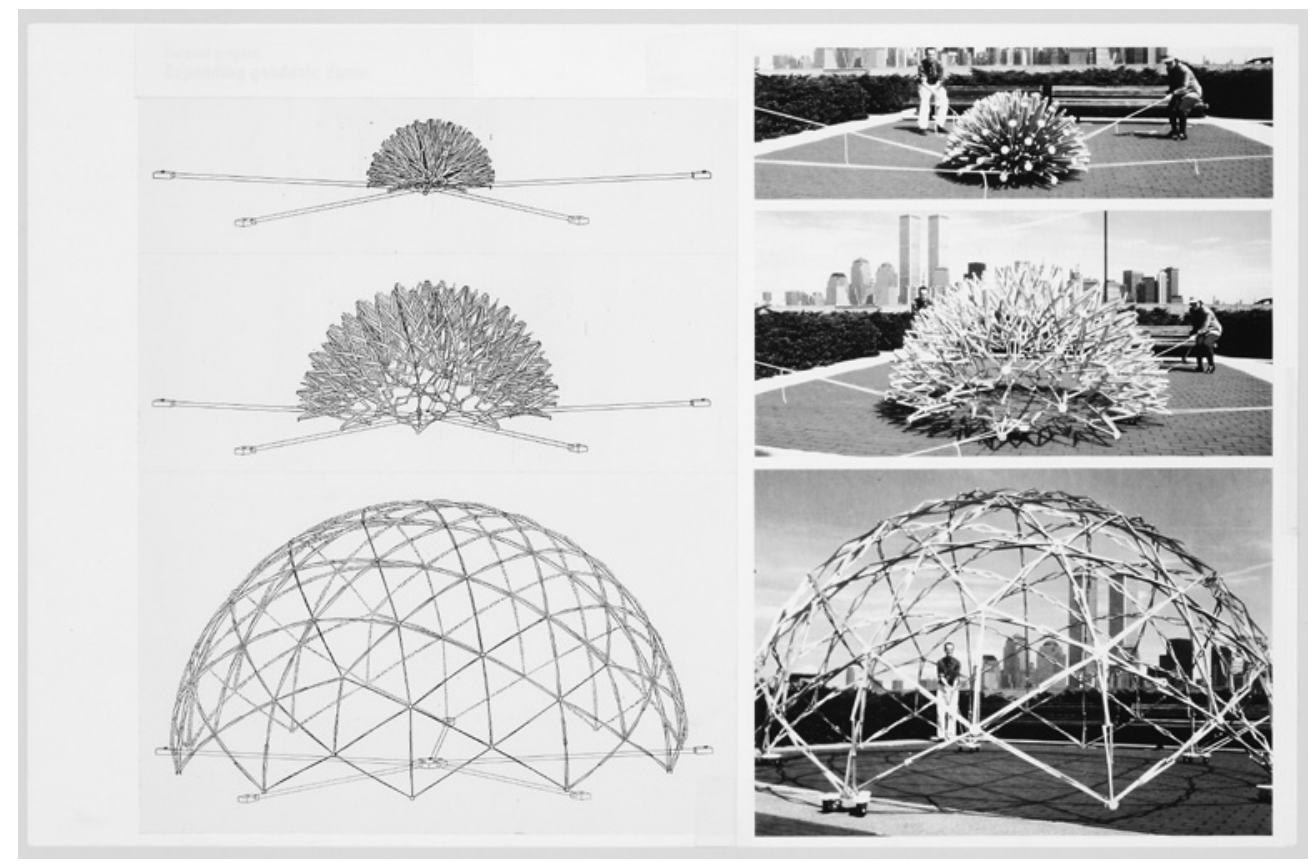

Chuck Hoberman, Hoberman Associates.

Dôme géodésique déployable, 1991. Chuck Hoberman fonds, Centre Canadien d'Architecture

(c) Hoberman Associates. Collage d'épreuves électrostatiques sur papier, $28 \times 43,2 \mathrm{~cm}$.

les nuances de l'aspect numérique de ces mutations ne peuvent être absorbées par le visiteur moins averti, il reste que la monstration des processus de transfert en quatre exemples et plus permet une lecture cohérente et aisée de cette histoire, L'Archéologie du numérique.

Le manque d'historicisation concernant l'apport du numérique dans la pratique de l'architecture a maintes fois été soulevé générant une réplique, selon les spécialistes, encore trop restreinte. Antonino Saggio avait lancé, il y a plus de dix ans, une série intitulée The IT Revolution in Architecture; Mario Carpo a publié récemment The Digital Turn in Architecture 1992-2012; il y a bien entendu The Autopoiesis of Architecture de Patrick Schumacher, Culture numérique et architecture: une introduction d'Antoine Picon, et quelques autres textes de référence importants. L'exposition de Greg Lynn, tout comme la publication du même nom qui accompagne l'exposition, viennent ainsi nourrir la réflexion et ouvrir de nouvelles avenues pour la recherche afin que puissent se consolider ces histoires de l'architecture numérique et devenir la trame d'un récit plus grand. 